\title{
Etiology and Epidemiological Conditions Promoting Fusarium Root Rot in Sweetpotato
}

\author{
A. C. Scruggs and L. M. Quesada-Ocampo
}

First author: Graduate Research Assistant, and second author: Assistant Professor, Department of Plant Pathology, North Carolina State University, Raleigh 27695-7616.

Accepted for publication 28 March 2016.

\begin{abstract}
Scruggs, A. C., and Quesada-Ocampo, L. M. 2016. Etiology and epidemiological conditions promoting Fusarium root rot in sweetpotato. Phytopathology 106:909-919.

Sweetpotato production in the United States is limited by several postharvest diseases, and one of the most common is Fusarium root rot. Although Fusarium solani is believed to be the primary causal agent of disease, numerous other Fusarium spp. have been reported to infect sweetpotato. However, the diversity of Fusarium spp. infecting sweetpotato in North Carolina is unknown. In addition, the lack of labeled and effective fungicides for control of Fusarium root rot in sweetpotato creates the need for integrated strategies to control disease. Nonetheless, epidemiological factors that promote Fusarium root rot in sweetpotato remain unexplored. A survey of Fusarium spp. infecting sweetpotato in North Carolina identified six

species contributing to disease, with $F$. solani as the primary causal agent. The effects of storage temperature $\left(13,18,23,29\right.$, and $\left.35^{\circ} \mathrm{C}\right)$, relative humidity $(80,90$, and 100\%), and initial inoculum level (3-, 5-, and 7-mmdiameter mycelia plug) were examined for progression of Fusarium root rot caused by $F$. solani and $F$. proliferatum on 'Covington' sweetpotato. Fusarium root rot was significantly reduced $(P<0.05)$ at lower temperatures $\left(13^{\circ} \mathrm{C}\right)$, low relative humidity levels $(80 \%)$, and low initial inoculum levels for both pathogens. Sporulation of $F$. proliferatum was also reduced under the same conditions. Qualitative mycotoxin analysis of roots infected with one of five Fusarium spp. revealed the production of fumonisin B1 by F. proliferatum when infecting sweetpotato. This study is a step toward characterizing the etiology and epidemiology of Fusarium root rot in sweetpotato, which allows for improved disease management recommendations to limit postharvest losses to this disease.
\end{abstract}

Sweetpotato (Ipomoea batatas) is an important crop around the world, particularly in developing countries, and currently ranks seventh among the most important food crops worldwide in production (FAOSTAT 2009). Being very high in vitamin A and other essential nutrients (Woolfe 1992), sweetpotato roots are used to combat nutritional disorders in some countries of Africa and Asia. Sweetpotato roots are also an important livestock feed in countries such as China (Scott 1992), and are increasingly being used for pet food in the United States (Bickers 2015). A recent, rapid increase in U.S. sweetpotato consumption shows the growing importance of this crop across the nation, primarily through valueadded products such as sweetpotato fries and chips (Johnson et al. 2015). North Carolina is the leading producer of sweetpotato in the United States and is responsible for over $40 \%$ of the nation's production, which is valued at over $\$ 260$ million (NCDA\&CS 2015).

Although sweetpotato production is limited by a variety of abiotic and biotic factors, plant disease is a significant threat to the industry worldwide (Clark et al. 2013). Fusarium root rot is a major postharvest disease of sweetpotato, particularly in the southeastern United States, where the majority of sweetpotato crops are grown. Fusarium root rot, also known as dry rot or end rot (Clark 1980), occurs primarily on roots that have been wounded in the harvesting or packing process, which facilitates infection (Clark 1980). The disease progresses relatively slowly and mostly occurs during longterm storage. Root rot lesions on sweetpotato are generally circular, with light and dark brown concentric rings that can extend into the

Corresponding author: L. M. Quesada-Ocampo; E-mail address: lmquesad@ncsu.edu

*The $\boldsymbol{e}$-Xtra logo stands for "electronic extra" and indicates that six supplementary tables are published online.

http://dx.doi.org/10.1094/PHYTO-01-16-0009-R

(C) 2016 The American Phytopathological Society root, often forming open cavities in the tissue. Enlarged lesions become dry and sunken, and white mycelia may be visible on the outside of the lesion or in the inner cavities (Clark et al. 2013).

Numerous Fusarium spp. have been reported to infect sweetpotato roots (Farr and Rossman 2006); however, Fusarium solani is considered the primary cause of Fusarium root rot (Clark et al. 2013) and, thus, the subject of most research conducted to date. Due to the lack of research and survey in this area, it is likely that additional Fusarium spp. also infect sweetpotato and have gone unreported. The Fusarium genus is notorious for its ability to produce secondary metabolites, many of which are toxic to both humans and animals (Desjardins 2006). This has resulted in a significant amount of research in susceptible crops such as wheat and corn. F. proliferatum and $F$. verticilloides, both producers of fumonisin and important pathogens of small grains, have also been shown to infect sweetpotato (da Silva and Clark 2013; Farr and Rossman 2006). The extent to which these pathogens cause disease in sweetpotato and their mycotoxin potential in such a crop is unknown. In addition, no research has been conducted examining the epidemiological conditions that promote or suppress Fusarium root rot caused by Fusarium spp., which may provide valuable insight for controlling this disease and limiting potential mycotoxin contamination.

A recent study suggests that $F$. solani may survive in sweetpotato roots as a fungal endophyte due to contact with infested soil in fields but fails to produce any root rot symptoms if environmental conditions are not favorable (da Silva and Clark 2013). Once conditions are favorable, the pathogen systemically infects the root and can then be transmitted through infected propagation material (da Silva and Clark 2013). Avoiding wounding during harvest and postharvest handling, as well as proper curing soon after harvest, are important steps to minimizing Fusarium root rot. Approximately 100 days after planting, sweetpotato roots are hand harvested after being plowed up and transferred to large storage containers. Roots are then immediately stored at $29^{\circ} \mathrm{C}$ and $85 \%$ relative humidity for 
5 to 7 days to be cured (Edmunds et al. 2008). This process allows for suberization and wound periderm formation to heal any wounds that occurred during harvest and protects the root from further decay (Artschwager and Starrett 1931). After curing, roots are typically stored long-term at $13^{\circ} \mathrm{C}$ and $85 \%$ relative humidity (Edmunds et al. 2008).

Currently, no sweetpotato cultivars exist with complete resistance to Fusarium root rot; however, cultivar susceptibility has been shown to vary, because some cultivars are more resistant than others (Clark et al. 1986). No studies to date have examined the susceptibility of 'Covington' sweetpotato to Fusarium root rot, which is the primary variety being grown in North Carolina. Although it is known that environmental conditions affect disease progression in other crops and pathosystems (Granke and Hausbeck 2010; Whiting et al. 2001), no specific factors influencing Fusarium root rot of sweetpotato have been identified. Establishing what temperature, humidity, and inoculum level can favor disease is needed to develop disease management strategies and avoid losses due to postharvest decay. Therefore, the purpose of this study was twofold: (i) to identify the diversity of Fusarium spp. that are infecting sweetpotato roots in North Carolina and determine their virulence and potential for mycotoxin production and (ii) to determine the effects of various temperatures $(13,18,26$, 29 , and $35^{\circ} \mathrm{C}$ ), relative humidity levels $(80,90$, and $100 \%)$, and inoculum levels (3-, 5-, and 7-mm-diameter mycelial plugs) that may occur throughout the postharvest life of a sweetpotato root on disease progression of Fusarium root rot. With this information, accurate recommendations can be made to growers and packers so that environmental storage conditions can be optimized to limit the amount of postharvest losses due to Fusarium root rot and reduce the potential mycotoxin contamination of sweetpotato.

\section{MATERIALS AND METHODS}

Sampling and isolation of Fusarium spp. infecting sweetpotato. Covington sweetpotato roots with suspected Fusarium infection were sampled from commercial packinghouses in North Carolina, representing 11 commercial sweetpotato farms and covering seven counties in eastern North Carolina (Supplementary Table S1). Covington was selected due to its large-scale production in commercial operations in North Carolina. Roots were placed in either a plastic crate or bag, transported back to the lab, and stored at $4^{\circ} \mathrm{C}$ until pathogen isolation.

To isolate Fusarium spp., infected roots were first rinsed in tap water and then surface sterilized by spraying until drench with $80 \%$ ethanol. Roots were then transferred to a biosafety cabinet and allowed to air dry. Following drying, isolations were performed by cutting out a small triangular section from the edge of the lesion and placing it onto a $100-\mathrm{mm}$ petri dish containing potato dextrose agar (PDA) amended with ampicillin at $20 \mathrm{mg} / \mathrm{liter}$ and rifampicin at $20 \mathrm{mg} /$ liter. Plates were incubated at $23^{\circ} \mathrm{C}$ under continuous light. Fusarium isolates grown on these plates were transferred to a sterile $60-\mathrm{mm}$ petri dish of PDA and allowed to grow at $23^{\circ} \mathrm{C}$ under continuous light. Isolates were then single spored by adding $100 \mu \mathrm{l}$ of sterile water onto the surface of an actively growing culture and then spreading the $100-\mu \mathrm{l}$ droplet onto a PDA plate. After $24 \mathrm{~h}$, a single, germinated spore was selected using a light microscope and transferred to another PDA plate. Isolates were stored as mycelia plugs in a $1.5-\mathrm{ml}$ tube with sterile water and a hemp seed at $4{ }^{\circ} \mathrm{C}$ for long-term preservation.

Molecular identification of Fusarium isolates. For DNA extraction of isolates, a 5-mm mycelia plug from an actively growing culture was transferred to a $250-\mathrm{ml}$ flask containing $50 \mathrm{ml}$ of potato dextrose broth and incubated for a period of 7 days, under light and gentle shaking on an Advanced Digital Shaker (VWR International, Radnor, PA). Tissue was collected using vacuum filtration and lyophilized in 1.5- $\mathrm{ml}$ tubes for $48 \mathrm{~h}$. Tissue was then disrupted by adding three glass beads to each tube and homogenized for $30 \mathrm{~s}$ using an Omni Bead Ruptor 24 (Omni International, Kennesaw, GA). DNA extractions were performed using a standard phenol-chloroform extraction protocol (Iowa State University PTF 2003).

Total DNA was used to amplify the internal transcribed spacer (ITS) region with primers ITS1 and ITS4 (White et al. 1990). All polymerase chain reactions (PCR) were performed in a T100 Thermocycler (Bio-Rad, Hercules, CA) with the following parameters: $94^{\circ} \mathrm{C}$ for $3 \mathrm{~min} ; 30$ cycles of $94^{\circ} \mathrm{C}$ for $30 \mathrm{~s}, 50^{\circ} \mathrm{C}$ for $30 \mathrm{~s}$, and $72^{\circ} \mathrm{C}$ for $1 \mathrm{~min}$; and $72^{\circ} \mathrm{C}$ for $5 \mathrm{~min}$. Samples were then held at $4^{\circ} \mathrm{C}$. The translation elongation factor (TEF) region was also amplified for all isolates using primers ef 1 and ef 2 (Geiser et al. 2004). These PCR were performed with the following parameters: $94^{\circ} \mathrm{C}$ for $3 \mathrm{~min} ; 30$ cycles of $94^{\circ} \mathrm{C}$ for $30 \mathrm{~s}, 53^{\circ} \mathrm{C}$ for $30 \mathrm{~s}$, and $72^{\circ} \mathrm{C}$ for $1 \mathrm{~min}$; and $72^{\circ} \mathrm{C}$ for $5 \mathrm{~min}$. Samples were then held at $4^{\circ} \mathrm{C}$.

PCR products were cleaned up using ExoSAP-IT (Affymetrix, Santa Clara, CA) and sequencing was carried out at the Genomic Sciences Laboratory, North Carolina State University, on a LifeTech 3730xl DNA Analyzer (Life Technologies, Carlsbad, CA) using the forward primer that was used to amplify the DNA. Electropherogram files from the LifeTech 3730xl DNA Analyzer were viewed for quality of reaction in FinchTV (Geospiza, Inc., Seattle) and sequence reactions were repeated if necessary. Sequences were compared against the National Center for Biotechnology Information GenBank database using BLAST (Altschul et al. 1990) and the Fusarium-ID Database (Geiser et al. 2004) for species identification.

Phylogenetic analysis. Sequences were aligned using ClustalWS (Larkin et al. 2007) in the Jalview 2.9.0 (Waterhouse et al. 2009) software package. Alignments were visualized using Jalview 2.9.0 and the sequence ends were manually trimmed to remove lowquality bases. The ITS and TEF datasets were concatenated to produce a total sequence of $952 \mathrm{bp}$ for each isolate. Haplotypes were identified using the haplotype block workflow in the Mobyle SNAP Workbench (Monacell and Carbone 2014). Identical sequences were collapsed into 42 haplotype groups (Table 1). In all, 398 variable sites across the concatenated dataset were identified and further phylogenic analysis was conducted using only these sites.

Maximum-likelihood phylogenetic analysis was performed using RAxML (Stamatakis et al. 2005) version raxmlHPC-SSE3 as part of the Mobyle SNAP Workbench (Monacell and Carbone 2014). Phylogenetic analysis of all 42 haplotypes was conducted using the maximum-likelihood method based on the GTRGAMMA substitution model, with a rapid bootstrap random number seed set at 2,139 . The algorithm selected performed a rapid bootstrap analysis and searched for the highest scoring maximum-likelihood tree in one run. In addition to maximum-likelihood analysis, maximum-parsimony analysis was also conducted in MEGA 6.06 (Tamura et al. 2013) using the subtree-pruning-regrafting algorithm (Nei and Kumar 2000) with search level 1, in which the initial trees were obtained by the random addition of sequences (10 replicates). Both analyses were conducted with gaps set as a fifth state, and clade stability was assessed using 500 bootstrap replications.

Virulence of Fusarium isolates. To verify the pathogenicity of each species identified in the survey and to assess the virulence of each species, one representative isolate was selected for infection assays in Covington sweetpotato (Table 2). Six Covington sweetpotato roots were wounded using a rubber-band-propelled wooden dowel previously developed by Holmes and Stange (2002), calibrated to inflict a bruising wound $2 \mathrm{~mm}$ deep into the sweetpotato root. Roots were inoculated using a 5-mm mycelia plug from each isolate. Two roots were placed per clear plastic chamber along with a paper towel dampened with $15 \mathrm{ml}$ of water to maintain high humidity (>95\%). Roots were stored in growth chambers set at $23^{\circ} \mathrm{C}$.

Roots were evaluated every other day for 11 days for lesion diameter by measuring the longest diameter of the lesion. The experiment was arranged in a randomized complete block design with two replications. Area under the disease progress curve (AUDPC) values were calculated for lesion diameter per root using the trapezoidal method (Madden et al. 2007). Data residuals were calculated, checked for normality using the Shapiro-Wilk normality test, Q-Q 
plots, and boxplots, and for equality of variances with Levene's test using R (R Core Team 2013). Once residuals were found to follow statistical assumptions, the data were subjected to analysis of variance (ANOVA) and effects were considered significant at 0.05 and means separated using Tukey's test.

Mycotoxin analysis. Three sweetpotato roots were wounded and inoculated as described above. Using 5-mm mycelia plugs, roots were inoculated with different Fusarium spp. identified in the survey that have been reported to produce deoxynivalenol (DON), T-2, or fumonisin B1 (Table 3). Two weeks after inoculation, infected sweetpotato tissue was collected from each root using a 7-mm cork borer. The infected tissue from the three roots was combined into a $50-\mathrm{ml}$ tube and stored at $-20^{\circ} \mathrm{C}$ until mycotoxin analysis.

Infected sweetpotato tissue was crushed using a mortar and pestle. Qualitative mycotoxin analysis was performed using a Lateral Flow Device Test Kit (AC Diagnostics, Fayetteville, AR) with a minimum detection limit of $200 \mathrm{ppb}$, following the manufacturer's instructions for each tested mycotoxin. Positive tests were repeated once with a different test kit and once in time with an additional infection series.

Host and pathogen material for epidemiological studies. Actively growing cultures of $F$. solani isolate AS086 and $F$. proliferatum isolate AS050 were used for inoculation in each experiment. Isolate AS086 was collected from an infected sweetpotato sample and isolate AS050 was collected from an infected garlic sample, both obtained from the North Carolina State Plant Disease and Insect Clinic and hyphal tipped to ensure pure cultures. DNA extraction followed by amplification of the ITS region using primers ITS4 and ITS5 (White et al. 1990) was performed and PCR products were sequenced to molecularly characterize the isolates. A BLAST search of the produced sequences from AS086 revealed a 100\% identity to GenBank accession number JF323000, which corresponded to an $F$. solani isolate. Likewise, sequences generated from isolate AS050 revealed a $100 \%$ identity to GenBank accession number KJ608097, corresponding to an $F$. proliferatum isolate. ITS sequences for the $F$. solani and $F$. proliferatum isolates used in this study were deposited into GenBank under accession numbers KU507202 and KU507203, respectively. Cultures were maintained on PDA and stored at $4{ }^{\circ} \mathrm{C}$. Isolates were transferred onto fresh PDA media 7 days before inoculation and kept under light at $23^{\circ} \mathrm{C}$.

TABLE 1. Diversity of Fusarium spp. from a survey of infected sweetpotato roots obtained from 11 commercial sweetpotato farms across seven counties in North Carolina

\begin{tabular}{lcc}
\hline Species & Number of isolates & Unique haplotypes \\
\hline F. solani & 54 & 16 \\
F. oxysporum & 49 & 20 \\
G. fujikuroi complex & 12 & 2 \\
F. acuminatum & 7 & 2 \\
F. incarnatum & 3 & 1 \\
F. graminearum & 1 & 1 \\
Total & 126 & 42 \\
\hline
\end{tabular}

TABLE 2. Average area under the disease progress curve (AUDPC) values observed 11 days postinoculation in sweetpotato roots infected with a single isolate of each species found in our survey

\begin{tabular}{llc}
\hline Isolate & \multicolumn{1}{c}{ Species } & Mean AUDPC $\pm \mathrm{SE}^{\mathrm{z}}$ \\
\hline AS311 & Gibberella fujikuroi complex & $39.38 \pm 4.70 \mathrm{a}$ \\
AS495 & Fusarium oxysporum & $78.25 \pm 8.41 \mathrm{~b}$ \\
AS419 & F. incarnatum & $107.38 \pm 4.29 \mathrm{bc}$ \\
AS494 & F. acuminatum & $113.83 \pm 9.19 \mathrm{c}$ \\
AS086 & F. solani & $148.67 \pm 7.79 \mathrm{~d}$ \\
AS256 & F. graminearum & $206.79 \pm 11.06 \mathrm{e}$ \\
\hline
\end{tabular}

${ }^{\mathrm{z}}$ Mean AUDPC \pm standard error (SE) calculated by measuring lesion diameter every other day for 11 days. Different letters represent statistical significance at the 0.05 significance level, with means separated using Tukey's test.
Covington roots were obtained from individual commercial sweetpotato farms at the time of each inoculation. All roots used in this study had been previously cured at industry standards (Edmunds et al. 2008) and were selected based upon consistent size, shape, and a disease-free appearance. Prior to inoculation, sweetpotato roots were surface sterilized by soaking them in $5 \%$ sodium hypochlorite for $5 \mathrm{~min}$, individually rinsing them with sterile water, and placing them into a laminar flow hood to air dry. Upon drying, sweetpotato roots were placed into a plastic box one at a time.

Wounding and inoculation. Prior studies indicate that bruising is the most conducive wound type for disease development (Holmes and Stange 2002). Therefore, in order to bruise roots, a 589-g metal rod with a diameter of $10 \mathrm{~mm}$, sterilized with $80 \%$ ethanol, was dropped through a $19 \mathrm{~mm}$ PVC pipe (to ensure accuracy) onto the middle of the sweetpotato from a height of $45 \mathrm{~cm}$, thus eliciting an average impact force of 866 Newtons $(\mathrm{N})$. A consistent bruising wound approximately 3 to $5 \mathrm{~mm}$ deep and $10 \mathrm{~mm}$ in diameter was inflicted on the roots. Roots were immediately placed individually, with the wounded side up, into sterile, clear, 28-by-20-by-14-cm plastic containers.

Sweetpotato roots were inoculated using mycelium plugs from 7-day-old cultures. Plugs were taken along the outer edge of cultures to ensure that the same amount and type of inoculum was contained in each plug. Plugs were placed face down onto the edge of the wounded area of the root, ensuring that the fungus contacted internal tissue beyond the periderm.

Effects of temperature and humidity on disease development. Six wounded roots inoculated with a 5-mm-diameter mycelium plug of $F$. solani or $F$. proliferatum and six wounded but not inoculated control roots were placed in individual containers and incubated in a growth chamber set at either $13,18,23,29$, or $35^{\circ} \mathrm{C}$. Humidity was maintained at 80,90 , and $100 \%$ for each temperature treatment to test for interaction effects between temperature and humidity. Preliminary experiments were performed to consistently achieve the desired humidity levels by adding specific volumes of water inside the container. The method was standardized at $23^{\circ} \mathrm{C}$ and took into account the humidity derived from the normal respiration of an uninfected root by placing a sweetpotato in the container during preliminary experiments. A level of $80 \%$ humidity was achieved by placing a sweetpotato alone into the chamber, while $90 \%$ humidity required the addition of $10 \mathrm{ml}$ of sterile water in a $60 \mathrm{~mm}$ petri dish and $100 \%$ humidity required the addition of three paper towels dampened with $15 \mathrm{ml}$ of sterile water. Watchdog model B102 data loggers (Spectrum Technologies, Plainfield, IL) were placed randomly in each treatment to monitor the temperature and relative humidity throughout the duration of the experiment (Table 4).

Roots were evaluated every other day for 11 days for lesion diameter by measuring the longest diameter of the lesion and for sporulation area by measuring the diameter of fungal growth on the root surface. The experiment was conducted twice and each of the six roots per experiment was an independent observation. AUDPC values were calculated for each variable per root using the

TABLE 3. Mycotoxin test results from sweetpotato roots infected with different Fusarium spp. 14 days postinoculation using lateral-flow test kits

\begin{tabular}{lllc}
\hline Isolate & \multicolumn{1}{c}{ Species } & Mycotoxin tested ${ }^{\mathrm{x}}$ & Result $^{\mathrm{y}}$ \\
\hline AS256 & Fusarium graminearum & Deoxynivalenol & - \\
PH-1 & F. graminearum & Deoxynivalenol & - \\
AS050 & F. proliferatum & Fumonisin B1 & + \\
AS135 & F. oxysporum & Fumonisin B1 & - \\
AS088 & F. acuminatum & T-2 & - \\
AS086 & F. solani & T-2 & - \\
\hline
\end{tabular}

$\bar{x}$ Mycotoxin tested was selected based on previous reports of being produced by the given Fusarium spp.

y Result is based on the reaction of a lateral-flow device with a minimum detection point of $200 \mathrm{ppb}$.

z $\mathrm{PH}-1$ = known deoxynivalenol producer in wheat (Gaffoor et al. 2005). 
trapezoidal method (Madden et al. 2007). The experiment was arranged in a split-plot design, with experiment as the blocking factor, temperatures as whole plots, and humidity levels as subplots. The AUDPC values of the two replicated experiments were used for statistical analyses. AUDPC data from control roots were removed from the data set prior to statistical analyses because no infection occurred and no symptoms developed. An ANOVA was performed to test the model AUDPC $=$ mean + temperature + humidity + temperature-humidity + error (experiment) using the aov function in R ( R Core Team, 2013). In the case of a significant interaction effect, a pairwise $t$ test was conducted to determine simple effects and $P$ values were adjusted using Holm's method.

Effect of temperature and inoculum level on disease development. Sets of six wounded roots inoculated with either 3-, 5-, or 7-mm-diameter mycelia plugs for each pathogen and six wounded but not inoculated control roots were placed into a growth chamber set at either $13,18,23,29$, and $35^{\circ} \mathrm{C}$. Roots were maintained at approximately $100 \%$ humidity using the methods described above. These roots were incubated for 11 days in the dark and Watchdog model B102 data loggers (Spectrum Technologies) were placed randomly in each treatment to monitor the temperature and relative humidity throughout the duration of the experiment. This experiment was conducted two times and disease evaluation and data analysis were performed as described in the temperature and humidity experiments, with inoculum level as the subplot.

\section{RESULTS}

Fusarium spp. survey. Alignment of the 123 ITS sequences resulted in a trimmed dataset of $405 \mathrm{bp}$ and alignment of the 123 TEF sequences resulted in a trimmed dataset of $547 \mathrm{bp}$, producing a concatenated dataset of $952 \mathrm{bp}$. In all, 81 isolates were identical in sequence and, therefore, collapsed to a single haplotype, resulting in 42 unique haplotypes with 398 variable sites that were used for phylogenic analysis. F. incarnatum isolates AS318 and AS343 and Gibberella fujikuroi complex isolate AS347 were excluded from the analysis due to failure of the elongation factor region to amplify. However, preliminary reconstruction of a phylogenic tree using neighbor joining based on ITS sequence alone showed isolates AS318 and AS343 cluster with isolate AS419, all three of which are identified as F. incarnatum. A phylogeny based on maximumlikelihood analysis was inferred and the tree with the highest likelihood is shown (Fig. 1, TreeBASE Study accession number S18706). Maximum-parsimony analysis was also performed for the 42 haplotypes (tree not shown) and 263 sites were parsimony informative. The most parsimonious tree had length $=579$, with a consistency index of 0.751012 , retention index of 0.956150 , and a composite index of 0.753030 and 0.718080 and for all sites and parsimony-informative sites, respectively.

A virulence test was conducted to ensure that each collected species was capable of reinfecting sweetpotato roots to produce symptoms similar to those observed. Isolates used to represent each species were all capable of infecting sweetpotato roots and reproduced dark lesions, characteristic of external symptoms Fusarium root rot. Although lesion depth was not measured in

TABLE 4. Mean relative humidities observed in the incubation chambers across all test temperature treatments over the course of each 11-day study on the progression of Fusarium root rot caused by Fusarium proliferatum and F. solani

\begin{tabular}{lcc}
\hline & \multicolumn{2}{c}{ Mean $\mathrm{RH}(\%) \pm \mathrm{SE}^{\mathrm{z}}$} \\
\cline { 2 - 3 } Goal RH $(\%)$ & F. proliferatum & F. solani \\
\hline 80 & $76.02 \pm 0.20$ & $77.39 \pm 0.28$ \\
90 & $93.15 \pm 0.12$ & $91.58 \pm 0.21$ \\
100 & $98.06 \pm 0.06$ & $97.09 \pm 0.07$ \\
\hline
\end{tabular}

$\mathrm{z}$ Mean relative humidity $(\mathrm{RH}) \pm$ standard error $(\mathrm{SE})$. roots infected with different species, the $F$. oxysporum isolate and the isolate from the G. fujikuroi species complex were confined primarily to the surface of the root and did not consistently extend below the periderm. Analysis of AUDPC values collected over an 11-day period revealed significant differences in disease progression between species. F. graminearum infection resulted in the highest disease level, followed by the typical root rot pathogen, $F$. solani (Table 2). The lowest level of disease occurred with the isolate used to represent the G. fujikuroi complex (Table 2). Furthermore, because all species identified in the survey had been previously reported to infect sweetpotato except $F$. graminearum, this species was reisolated from infected roots to confirm $F$. graminearum as the causal agent.

Mycotoxin analysis. Mycotoxin analysis was performed using lateral-flow devices for an infection series of roots inoculated with one of five Fusarium spp. (Table 3). F. graminearum isolate AS256 was collected from an infected sweetpotato root and tested negative for DON at a minimum detection concentration of $200 \mathrm{ppb}$. PH-1, an $F$. graminearum isolate known to produce DON in cereal crops (Gaffoor et al. 2005), also tested negative at $200 \mathrm{ppb}$ when inoculated onto a sweetpotato. $F$. proliferatum, which was previously reported on sweetpotato (da Silva and Clark, 2013) and is a well known producer of fumonisin B1, tested positive for fumonisin at a 200-ppb detection limit. A second infection series with this pathogen was generated and subjected to an additional fumonisin B1 test, in which it once again was positive at the 200-ppb detection limit. F. acuminatum is a known producer of T-2 toxin in other crops and has been previously reported to infect sweetpotato (Farr and Rossman 2006). A test for T-2 toxin in infected sweetpotato roots with a minimum cutoff of $200 \mathrm{ppb}$ proved negative. $F$. solani and F. oxysporum, the two most common species associated with rots of sweetpotato, tested negative for T-2 and fumonisin B1, respectively (Table 3).

Effect of temperature, humidity, and inoculum level on disease. Symptoms consistent with Fusarium root rot as described by Clark et al. (2013), entailing dry, dark, sunken lesions that extended beyond the root periderm, were observed at each temperature, relative humidity level, and inoculum level tested. Advanced lesions formed under optimal conditions contained hollow cavities under the lesion surface, with white mycelia protruding from the cavities (Fig. 2). Symptoms of Fusarium root rot were delayed at $13^{\circ} \mathrm{C}$, not appearing until 4 days postinoculation (dpi) for $F$. solani as compared with 2 dpi for the other temperature treatments, across all levels of relative humidity and inoculum level. Similar results were observed for $F$. proliferatum, with the exception of the high inoculum level treatment (7-mm-diameter plug), which also resulted in symptom development at $2 \mathrm{dpi}$. Across all levels of temperature, disease symptoms were observed at 2 dpi for all levels of relative humidity and inoculum level. However, the $80 \%$ humidity level resulted in only a few instances of disease this early after inoculation, with most symptoms being observed beginning at $4 \mathrm{dpi}$ for both $F$. solani and $F$. proliferatum. In general, changes in temperature affected both time of symptom development and rate of disease progression, whereas changes in relative humidity largely affected the rate of disease progression. Changes in inoculum level affected disease severity at each time point measured for both pathogens.

No consistent sporulation was observed at any treatment with $F$. solani. Sporulation of $F$. proliferatum on infected tissue was observed at each temperature at 2 dpi across all levels of relative humidity and inoculum level treatments. Furthermore, sporulation was typically delayed until at least 4 dpi for most inoculated roots at $80 \%$ humidity. Sporulation at humidities of 90 and $100 \%$ were commonly observed at 2 dpi across most temperature treatments. No sporulation was observed at 80 or $90 \%$ humidity for $F$. proliferatum inoculated roots stored at $35^{\circ} \mathrm{C}$. Inoculum level differences typically affected the amount of sporulation rather than a delay in its development.

Disease progression was highest for $F$. solani at $29^{\circ} \mathrm{C}$ and F. proliferatum at $23^{\circ} \mathrm{C}$ compared with other tested temperatures 
under high humidity. Both pathogens also resulted in higher disease levels at $100 \%$ relative humidity and high inoculum levels (7-mmdiameter plug). Sporulation of $F$. proliferatum was also highest at $23^{\circ} \mathrm{C}, 100 \%$ humidity, and the high inoculum level (7-mm-diameter plug). The lowest levels of disease progression were observed at temperatures of $13^{\circ} \mathrm{C}$, relative humidities of $80 \%$, and low inoculum levels (3-mm plug) for both pathogens (Fig. 3). Significant interactions occurred between temperature and humidity $(P<0.0001)$ for lesion diameter AUDPC values for both $F$. solani and $F$. proliferatum. The interaction between temperature and inoculum level was also significant $(P<0.001)$ for $F$. proliferatum. Interactions between temperature and humidity and temperature and inoculum level were also significant $(P<0.0001)$ for sporulation of $F$. proliferatum. No interaction between temperature and inoculum level for $F$. solani revealed a significant main effect $(P<0.001)$ of inoculum level on disease progression, because AUDPC increased with inoculum level at each tested temperature (Fig. 3E and G).

AUDPC values for lesion diameter at $80 \%$ humidity stayed relatively constant across all tested temperatures for $F$. solani (Fig. 3A) and no significant simple effects of temperature were observed at the $80 \%$ humidity level (Supplementary Table S2). A level of $90 \%$ humidity produced significantly higher disease levels for $F$. solani at 18,23 , and $29^{\circ} \mathrm{C}$ compared with the same temperatures at $80 \%$ humidity; however, maximum disease levels at each temperature were observed at $100 \%$ humidity, except at temperatures of $13^{\circ} \mathrm{C}$ for F. solani (Fig. 3A). At $13^{\circ} \mathrm{C}$, there was no significant difference in disease progression of $F$. solani observed regardless of the humidity level. However, as temperatures became more favorable for $F$. solani disease development $\left(18,23,29\right.$, and $\left.35^{\circ} \mathrm{C}\right)$, a positive relationship between AUDPC and relative humidity level was observed (Fig. 3C), leading to increased disease progression at higher humidity levels. At 18,23 , and $29^{\circ} \mathrm{C}$, simple effects of relative humidity were significant at each of the tested humidity levels.

Similarly to $F$. solani, disease progression for $F$. proliferatum at $80 \%$ humidity remained relatively constant across temperature treatments (Fig. 3B) and revealed no significant simple effects between the tested temperatures (Supplementary Table S3). At the $90 \%$ humidity level, significant differences were observed between 13 and $29^{\circ} \mathrm{C}$ and 18 and $29^{\circ} \mathrm{C}$. The highest disease levels for $F$. proliferatum occurred at the $100 \%$ humidity level for each temperature besides $13^{\circ} \mathrm{C}$ (Fig. 3D), at which there were no significant differences between humidity levels. Humidity is significant between the 90 and $100 \%$ and the 80 and $100 \%$ treatments at each of the other temperature levels $\left(18,23,29\right.$, and $\left.35^{\circ} \mathrm{C}\right)$. At the $100 \%$ humidity level, $F$. proliferatum disease progression at $23^{\circ} \mathrm{C}$ was significantly higher than at any other tested temperature.

Unlike $F$. solani, F. proliferatum also had a significant interaction between temperature and inoculum level. The highest AUDPC values were observed at $23^{\circ} \mathrm{C}$ with the high inoculum level (7-mm) and the lowest AUDPC values were observed at $13^{\circ} \mathrm{C}$ with the low inoculum level (3-mm) (Fig. 3F). In general, there was a positive relationship between AUDPC and inoculum level (Fig. $3 \mathrm{H}$ ) because the high $(7-\mathrm{mm})$ inoculum level was significantly different from the low (3-mm) inoculum level at each tested temperature (Supplementary Table S4). The medium (5-mm) inoculum level varied at each temperature in its difference from the other tested inoculum levels (Fig. 3F). Significant differences in temperature were observed at each level of inoculum.

Similar observations were made for sporulation of $F$. proliferatum over the same treatments (Fig. 4). The highest sporulation was observed at $100 \%$ humidity for temperature treatments of 18,23 , and $29^{\circ} \mathrm{C}$, which were not significantly different (Supplementary Table S5). Sporulation generally then decreased at each of those temperatures as humidity levels dropped (Fig. 4A). Sporulation area at $13^{\circ} \mathrm{C}$ was significantly higher at $90 \%$ humidity as compared with 80 and $100 \%$ humidity levels and sporulation was nonexistent at $35^{\circ} \mathrm{C}$ across all humidity treatments (Fig. 4B). Significant interactions were also observed for $F$. proliferatum sporulation between temperature and inoculum level. As temperature approached $18^{\circ} \mathrm{C}$, high inoculum levels (7-mm plug) had the most sporulation (Fig. 4C), significantly higher than high inoculum levels at other temperatures and the other tested inoculum levels at $18^{\circ} \mathrm{C}$ (Supplementary Table S6). At 23 and $29^{\circ} \mathrm{C}$, however, high and moderate (5-mm plug) inoculum levels resulted in similar levels of sporulation and the low (3-mm) inoculum level was significantly lower. A positive relationship for sporulation was observed between inoculum level and temperature at each temperature treatment, with the exception of $35^{\circ} \mathrm{C}$, at which sporulation was inconsistent (Fig. 4D). Sporulation was significantly lower at $35^{\circ} \mathrm{C}$ compared with the other tested temperatures for the medium and high inoculum levels.

\section{DISCUSSION}

Investigations into the diversity of Fusarium spp. infecting sweetpotato storage roots in North Carolina identified six species, with $F$. solani being recovered the most frequently. F. solani is known to be the primary causal agent of Fusarium root rot (Clark et al. 2013); however, numerous others have also been reported to occur (Farr and Rossman 2006). Information from such surveys can provide valuable insight into the populations of causal agents contributing to disease, resulting in better long-term control of disease. For example, in a similar survey of Fusarium spp. infecting potato, Hanson et al. (1996) found that the species of Fusarium causing disease depended on numerous factors, including whether tubers are used for seed, table stock, or processing; isolation year and location; and method of isolation. Furthermore, understanding a baseline population allows for future observations in population shifts that may be associated with control failure. Previous studies in dry rot of potato observed an increase in $F$. sambucinum as a causal agent due to its adaption of fungicide resistance, whereas $F$. solani had traditionally been the more commonly isolated species (Hanson et al. 1996). Although the current study investigates only the Fusarium spp. causing disease in the roots, recent observations demonstrating the ability of $F$. solani to survive as an endophyte in other plant parts (da Silva and Clark 2013) suggests that future work should address the endophytic potential of other species so that a better understanding of complex etiologies and interactions can be developed.

Of 126 isolates obtained from the conducted survey, 42 unique haplotypes were identified based on a 952-bp dataset of combined ITS and TEF sequences. Phylogenic analysis of the 398 variable sites among the combined dataset using maximum likelihood resulted in relationships among haplotypes (Fig. 1). However, previous phylogenies developed based upon combined rDNA cluster, B-tub, and elongation factor sequences of multiple species within the Fusarium genus that produced similar results based on maximum likelihood placed $F$. graminearum, $F$. incarnatum, and $F$. acuminatum further related from $F$. solani (Watanabe et al. 2011). These differences could be due to different loci sequenced in the present study or reduced resolution by basing the phylogeny on fewer loci. No study, to date, has examined the phylogeny among related Fusarium spp. in sweetpotato. Based on the results of the study at hand, it is apparent that there is genetic diversity among species as well as within the species of $F$. oxysporum and $F$. solani found infecting sweetpotato. Some haplotypes of $F$. solani and $F$. oxysporum were found in a single location, while other were found across multiple counties (Fig. 1). This suggests that certain haplotypes may be better adapted to infect sweetpotato roots, are more commonly distributed in the soil, or are being moved around multiple counties through infected seed or contaminated equipment. Bootstrap support for some branches within the $F$. solani and $F$. oxysporum clades is low $(<70)$. Investigations into the $F$. solani species complex by O'Donnell et al. (2008) also resulted in low bootstrap support in inferred phylogenies, suggesting the potential for cryptic speciation within the group. Such speciation has been reported in related fungi (Geiser et al. 1998) and, therefore, may be 
the cause of the homoplasy observed in the present study with $F$. solani and $F$. oxysporum; however, further studies should be conducted before such conclusions are drawn.

To confirm pathogenicity of the collected isolates and determine relative levels of virulence among species, a representative isolate of each species was used in a virulence assay. All species collected in the survey infected sweetpotato roots upon inoculation and produced root-rot-like symptoms. Under the tested conditions, $F$. graminearum infection resulted in the highest level of disease, followed by F. solani. To our knowledge, this is the first report of $F$. graminearum infecting sweetpotato storage roots. Based on the results of the epidemiological experiments conducted in the present study, the conditions used for the virulence assay may have been optimal for $F$. graminearum, while not being optimal for other species. Furthermore, infection rates and disease progression of some Fusarium spp. may also depend on the inoculation method, potentially affecting the outcome of this test as well, because method of wounding has been shown to affect disease levels of other sweetpotato pathogens (Holmes and Stange 2002). Similar results were observed in potato, where differences in tuber susceptibility varied among Fusarium spp. (Hanson et al. 1996). Regardless, the present findings highlight the potential of other Fusarium spp. besides $F$. solani to cause significant losses in sweetpotato.

In many developing countries, sweetpotato roots are an important feed for livestock (Scott 1992). Numerous cases in history exist reporting the toxicosis or death of various livestock after the ingestion of moldy sweetpotato (Peckham et al. 1972; Wilson 1973). However, this has largely been attributed to the phytotoxin ipomeanol, which induces lung edema in cattle and is produced by sweetpotato in response to pathogen attack (Boyd and Wilson 1972). To our knowledge, no reports of mycotoxin production during Fusarium infection of sweetpotato have been published. A qualitative mycotoxin assessment performed with the species associated with root rot of sweetpotato in this study revealed fumonisin B1 production by $F$. proliferatum but not by $F$. oxysporum. Fumonisin B1 commonly contaminates cereals and grains (Desjardins 2006) and can cause severe toxicosis in livestock, such as equine leukoencephalomalacia, lung edema, and pulmonary edema in swine (Ross et al. 1990). The reports of lung edema in cattle after being fed moldy sweetpotato are consistent with our findings of fumonisin B1

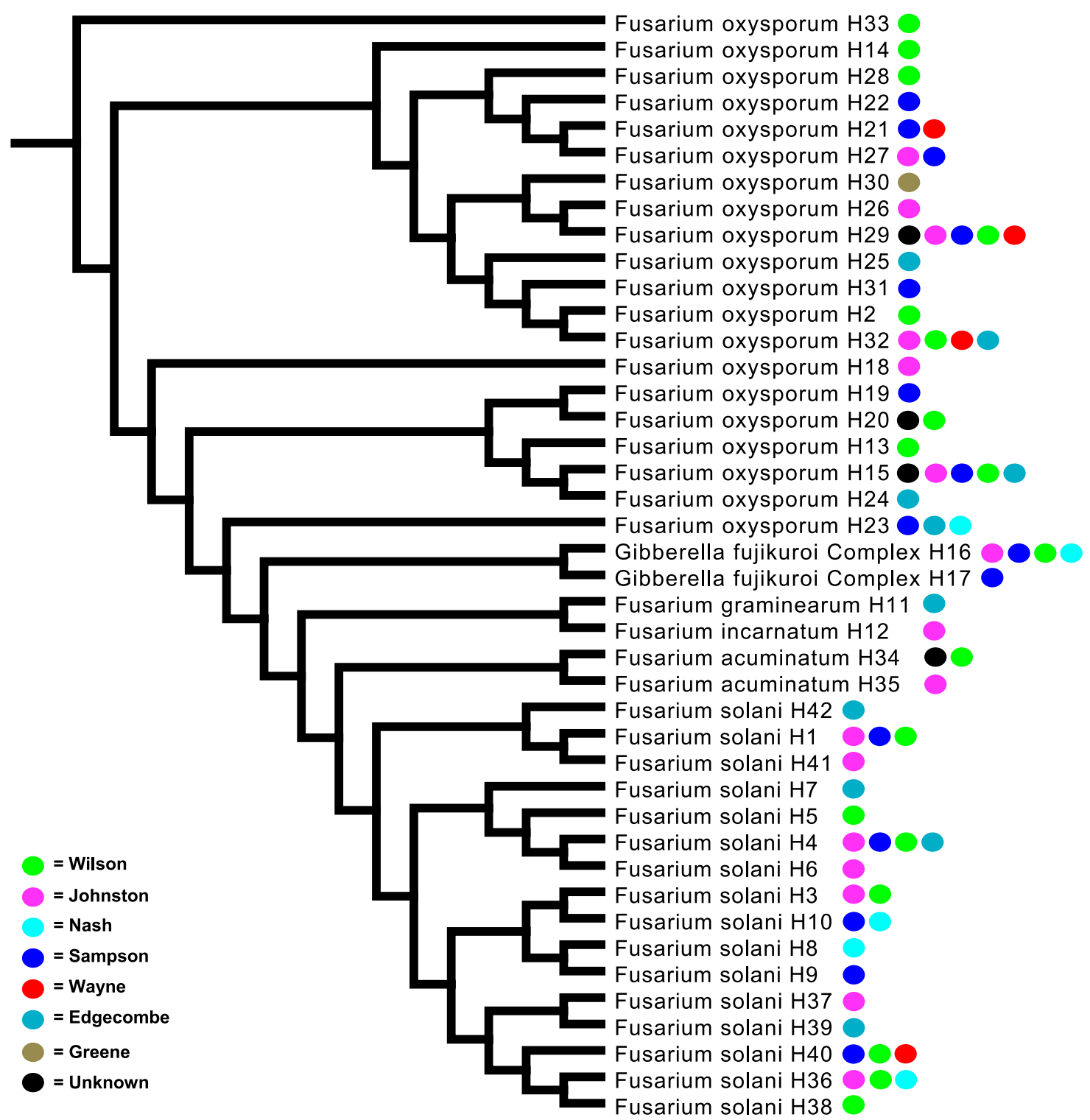

Fig. 1. Evolutionary history of the 42 unique haplotypes was inferred by using the maximum-likelihood method based on the GTRGAMMA substitution model. Maximum-likelihood phylogenetic analysis was performed using RAxML (Stamatakis et al. 2005) version raxmlHPC-SSE3 as part of the Mobyle SNAP Workbench (Monacell and Carbone 2014). The algorithm selected performed a rapid bootstrap analysis and searched for the highest scoring maximum-likelihood tree in one run, with a rapid bootstrap random number seed of 2,139 . Colored dots beside each haplotype represent the various counties in which the haplotype was found. 
in infected roots. Although lung edema has previously been associated with ipomeanol poisoning, based on the current findings, the potential of fumonisin contamination being an agent of disease cannot be discarded. No T-2 toxin, which is produced by $F$. solani (Ueno et al. 1975) and F. acuminatum (Rabie et al. 1986), was detected. Infection of sweetpotato by $F$. graminearum (which, to our knowledge, has not been previously reported) was also determined in this study. F. graminearum is notorious for contaminating grain crops with DON (Desjardins 2006); however, no DON production was detected in sweetpotato infected with $F$. graminearum isolate (AS256) and an isolate from cereals (PH-1) (Gaffoor et al. 2005). Although, here, we report the production of fumonisin B1 by $F$. proliferatum and negative results for other tested toxins, it is important to consider that only one isolate of each pathogen was used in our analysis and a limited set of potential mycotoxins was tested. Additionally, mycotoxin tests were conducted 2 weeks after inoculation, which may not have been enough time to allow for sufficient toxin accumulation in the sweetpotato tissue for detection. For example, in corn, Miller et al. (1983) found that DON accumulation was highest at 7 weeks after inoculation with $F$. graminearum, and significant levels of zearalenone were not detected until 9 weeks after inoculation with the same pathogen. Furthermore, mycotoxin production can vary by isolate or environmental factors, and the investigated species also produce additional toxins that were not tested. The study at hand introduces the potential for mycotoxin production in sweetpotato but additional studies are certainly needed to perform more quantitative analyses using multiple isolates and to test additional toxins that could be produced, especially by common species causing root rot of sweetpotato before we can understand the risk associated with using infected sweetpotato roots as livestock or pet food.

The current study quantifies the disease level and progression of Fusarium root rot in sweetpotato by $F$. solani and $F$. proliferatum at different storage conditions. Differences in disease progression at certain storage conditions between $F$. solani and $F$. proliferatum, as highlighted in this study, suggests the role that storage conditions may play in providing a fitness advantage of one Fusarium spp. over another. A study by Reid et al. (1999) found that certain temperatures within corn silks favored growth of $F$. moniliforme over that of $F$. graminearum and other temperatures favored growth of $F$. graminearum over F. moniliforme in mixed inoculations. Similarly, the species responsible for Fusarium head blight of wheat largely depends on climatic conditions within a geographic region, suggesting that the primary causal agent of a disease can differ depending on environmental conditions (Doohan et al. 2003). The extent to which field and storage conditions may affect the fitness or interaction of Fusarium spp. infecting sweetpotato is unknown. The current study demonstrates the effects of storage conditions on infection by a single causal agent but future work in sweetpotato could consider the effects of these conditions on the interaction between species and its impact on diseases caused by complex etiologies. Based on the effects that environmental conditions have on other diseases, as

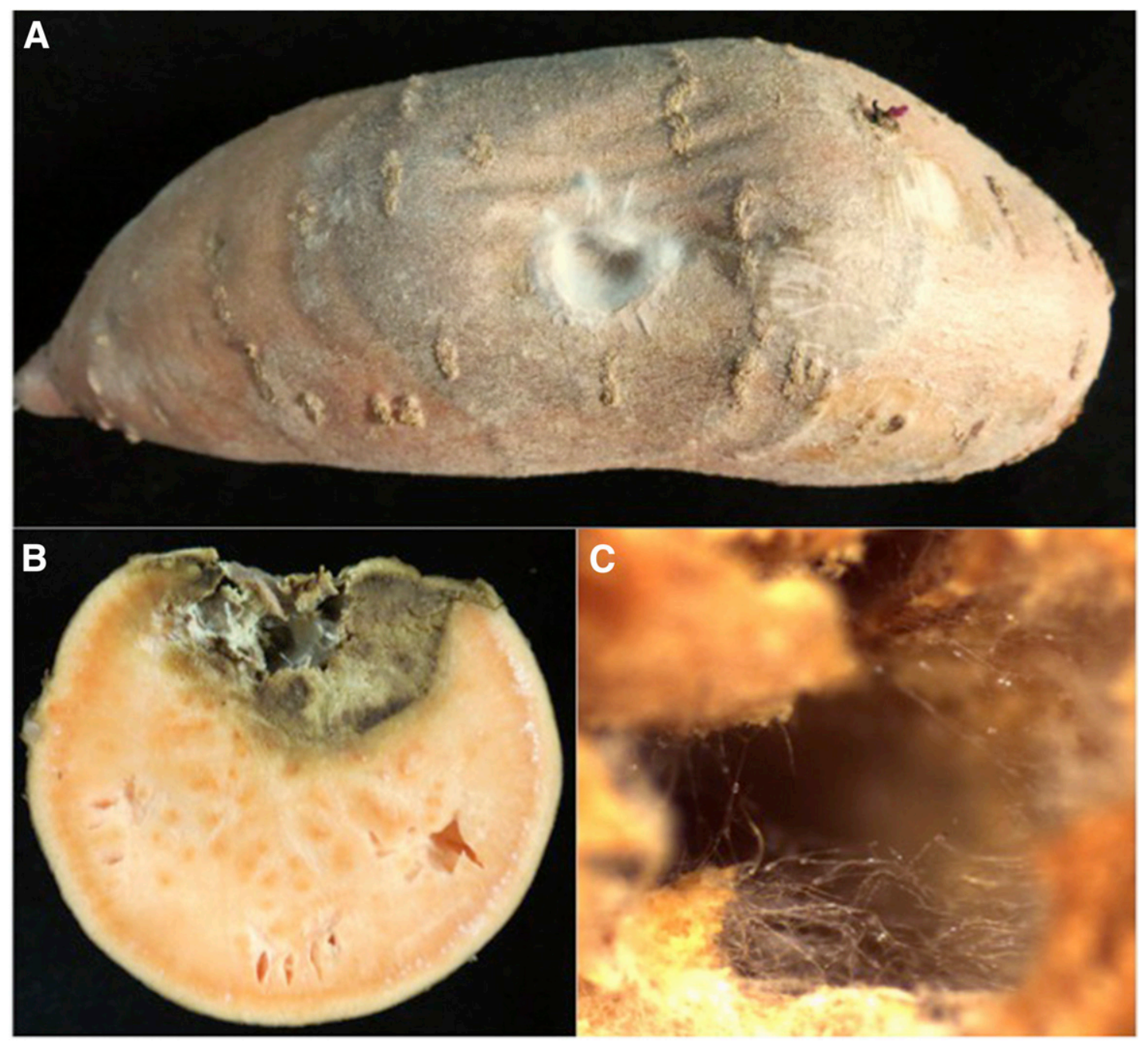

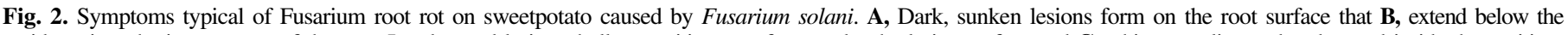
periderm into the inner cortex of the root. In advanced lesions, hollow cavities may form under the lesion surface and $\mathbf{C}$, white mycelia can be observed inside the cavities. 
highlighted above, and the observed differences between $F$. solani and $F$. proliferatum in sweetpotato at varying storage conditions, it may be possible that modifying these conditions will result in the increase or decrease of occurrence for particular species in sweetpotato. Furthermore, the diversity of Fusarium spp. infecting sweetpotato roots may also be affected by climatic conditions, such that certain species in warmer climates may be more prevalent there than in cooler climates, as seen in other cropping systems (Doohan et al. 2003).

Maximum disease levels produced by $F$. solani were observed at $29^{\circ} \mathrm{C}$, compared with $23^{\circ} \mathrm{C}$ for $F$. proliferatum, and temperatures
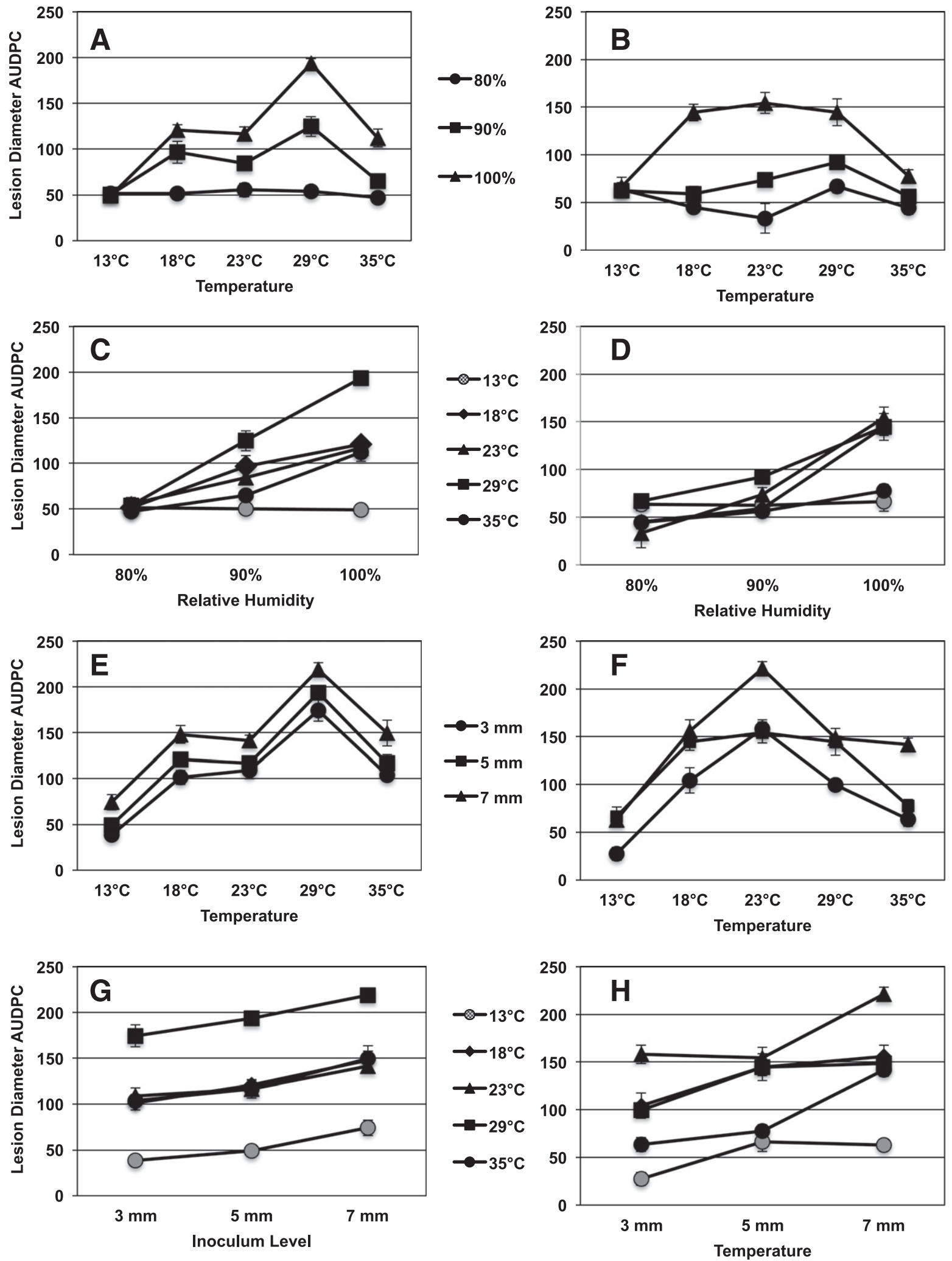

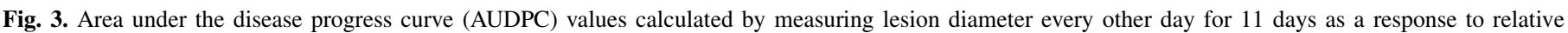

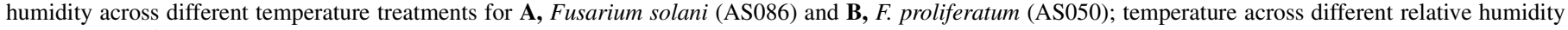

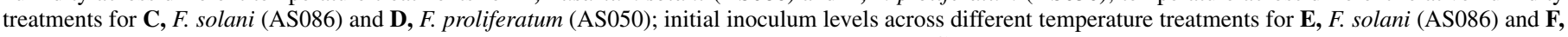

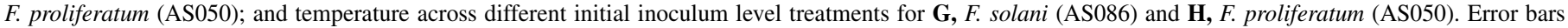
depict the standard error of the mean. 
above these levels resulted in a decrease in disease progression. High relative humidity levels $(100 \%)$ and high initial inoculum levels (7-mm) also resulted in significantly higher levels of disease for both pathogens. In general, lower initial inoculum levels can reduce disease levels further at each temperature. Previous studies on $F$. proliferatum identified isolates that grew at a temperature range of 4 to $35^{\circ} \mathrm{C}$, with optimal growth at $25^{\circ} \mathrm{C}$. No growth was observed over $35^{\circ} \mathrm{C}$ (Marin et al. 1995). Optimal in vitro growth of F. solani was found to be $30.5^{\circ} \mathrm{C}$ in a separate study (Goktepe et al. 2007). The differences in optimal in vitro growth between $F$. proliferatum and $F$. solani correspond to the differences in disease progression of root rot observed in this study, with $F$. solani causing higher levels of disease at higher temperatures compared with $F$. proliferatum. Differences in optimal infection temperatures for two species causing Fusarium head blight of wheat (F. graminearum and F. culmorum) have also been observed (Rossi et al. 2001). Temperature may also be important in limiting potential mycotoxin contamination, because Alberts et al. (1990) identified $25^{\circ} \mathrm{C}$ to be the optimal temperature for fumonisin production by $F$. moniliforme, with toxin levels decreasing at both lower and higher temperatures. Similar studies in dry rot of potato found that infection of tubers by F. sambucinum is greatest at $25^{\circ} \mathrm{C}$ but storage at lower temperatures $\left(<12^{\circ} \mathrm{C}\right)$ can result in slower progression of the disease (Lui and Kushalappa 2002). However, trichothecenes can still be produced in potato tubers at low temperatures (Leach and Webb 1981). Progression of Fusarium root rot decreased as relative humidity decreased for both pathogens. Previous studies of Fusarium head blight of wheat observed similar results, because infection of wheat spikes by $F$. avenaceum and $F$. graminearum was lower at lower compared with higher humidities (Rossi et al. 2001). However, infection by F. culmorum was higher at lower humidities, suggesting a differential response to humidity depending on the pathogen (Rossi et al. 2001).

In summary, Fusarium root rot can occur over a wide range of temperatures, relative humidities, and initial inoculum levels. Interactions between temperature and relative humidity for both $F$. solani and $F$. proliferatum reveal that storage at $13^{\circ} \mathrm{C}$ can have a similar effect in reducing disease progression regardless of the relative humidity. Likewise, storage at $80 \%$ humidity can reduce disease levels to a similar level regardless of temperature. Storage of sweetpotato roots at $80 \%$ humidity, however, is not recommended due to losses from physiological processes such as increased respiration (Ezell et al. 1956). Regardless of the species causing Fusarium root rot of sweetpotato, based on the results of this study, long-term storage of sweetpotato roots at $13^{\circ} \mathrm{C}$ and 85 to $90 \%$ humidity and maintaining sanitary conditions is recommended for reducing losses to Fusarium root rot. Current recommendations of storage at $13^{\circ} \mathrm{C}$ and $85 \%$ humidity with sanitization seem appropriate (Edmunds et al. 2008). Reducing initial inoculum levels by removing diseased roots from fields and packinghouses as well as maintaining sanitary harvest and packing equipment can also reduce levels of Fusarium root rot. F. solani and $F$. proliferatum responded differently to different storage temperatures, suggesting that certain conditions may favor one species over another. These differences may imply that the frequency and diversity of observed species in sweetpotato could differ geographically due to contrasting climatic conditions or that production practices such as curing at high temperatures may benefit the predominance of certain species. The confirmation of fumonisin B1 production by $F$. proliferatum demonstrates the potential for Fusarium spp. to produce mycotoxins in infected sweetpotato, and storage at the appropriate conditions identified in this study may help reduce potential mycotoxin contamination. A survey to determine the diversity of species that infect sweetpotato in North Carolina revealed six species causing disease, some of which are toxigenic. A more indepth population analysis of the Fusarium spp. identified in sweetpotato should be conducted to better understand the pathogen populations and interactions occurring on sweetpotato in North
Carolina. Although only F. proliferatum was confirmed to produce a mycotoxin in sweetpotato, further work is needed to quantify the amount being produced and also to test for additional toxins being produced by other species. Understanding the etiology and
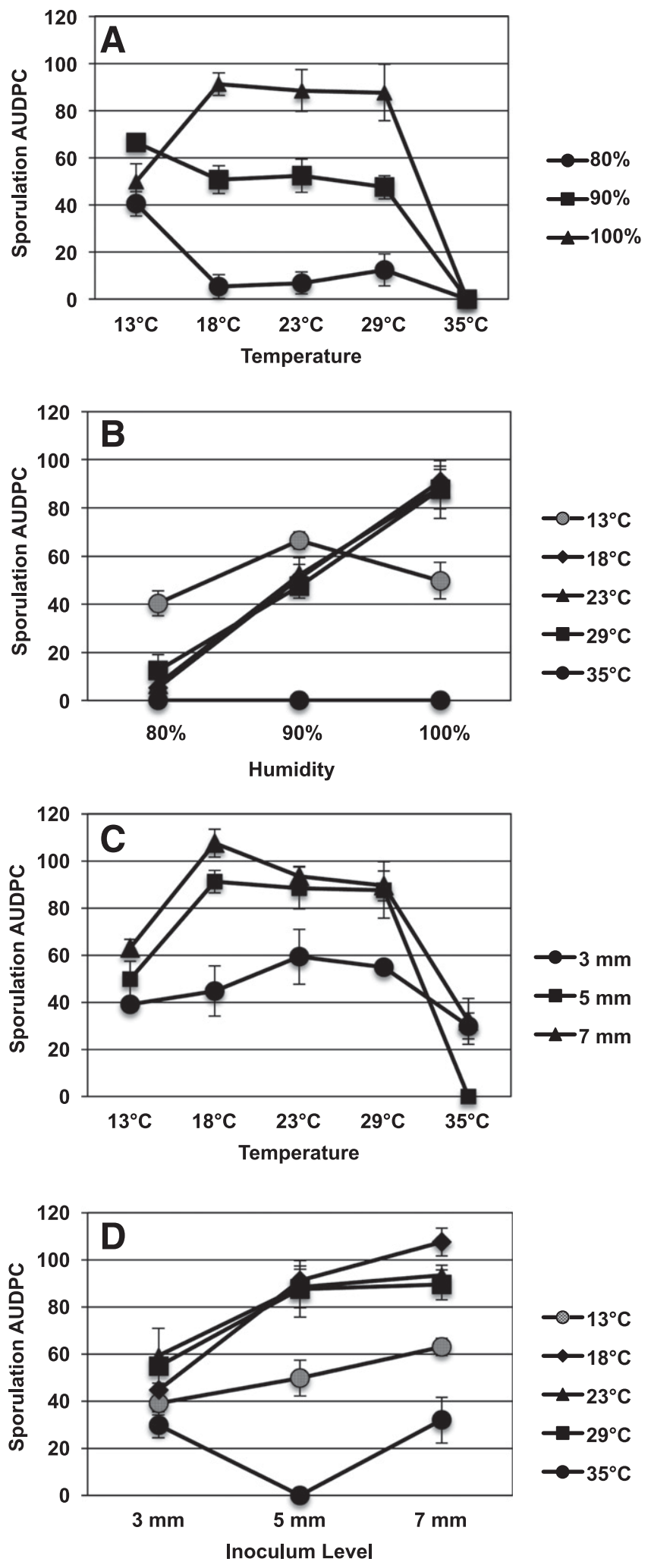

Fig. 4. Area under the disease progress curve (AUDPC) values calculated by measuring sporulation of Fusarium proliferatum every other day for 11 days as a response to $\mathbf{A}$, relative humidity across different temperatures; $\mathbf{B}$, temperature across different relative humidity treatments; $\mathbf{C}$, initial inoculum levels across different temperatures; and $\mathbf{D}$, temperature across different initial inoculum level treatments. Error bars depict the standard error of the mean. 
epidemiology of Fusarium root rot in sweetpotato can lead to improved control and management of this disease.

\section{ACKNOWLEDGMENTS}

This work was supported by funds from the North Carolina State University (NCSU) Hatch Project Number NC02418, the Golden Leaf Foundation, and the Bill and Melinda Gates Foundation. We thank all the members of the Quesada lab for their valuable help; S. Butler from the NCSU Plant Disease and Insect Clinic as well as F. Trail for contributing isolates that were used in the study; L. Dong for statistical support; and the numerous sweetpotato farms in North Carolina that allowed us to sample diseased roots.

\section{LITERATURE CITED}

Alberts, J. F., Gelderblom, W. C., Thiel, P. G., Marasas, W. F., Van Schalkwyk, D. J., and Behrend, Y. 1990. Effects of temperature and incubation period on production of fumonisin B1 by Fusarium moniliforme. Appl. Environ. Microbiol. 56:1729-1733.

Altschul, S. F., Gish, W., Miller, W., Myers, E. W., and Lipman, D. J. 1990. Basic local alignment search tool. J. Mol. Biol. 215:403-410.

Artschwager, E. F., and Starrett, R. C. 1931. Suberization and wound-periderm formation in sweet potato and gladiolus as affected by temperature and relative humidity. J. Agric. Res. 43:353-364.

Bickers, C. 2015. Sweet potatoes: Juice, pet food products could increase market demand. Online publication. Southeast Farm Press. http:// southeastfarmpress.com/vegetables/sweet-potatoes-juice-pet-food-productscould-increase-market-demand

Boyd, M. R., and Wilson, B. J. 1972. Isolation and characterization of 4ipomeanol, a lung-toxic furanoterpenoid produced by sweet potatoes (Ipomoea batatas). J. Agric. Food Chem. 20:428-430.

Clark, C. 1980. End rot, surface rot, and stem lesions caused on sweet potato by Fusarium solani. Phytopathology 70:109-112.

Clark, C., Randle, W., and Pace, C. 1986. Reactions of sweet potato selections to Fusarium root and stem canker caused by Fusarium solani. Plant Dis. 70: 869-871.

Clark, C. A., Ferrin, D., Smith, T., and Holmes, G. 2013. Compendium of Sweetpotato Diseases, Pests, and Disorders. American Phytopathological Society, St. Paul, MN

da Silva, W. L., and Clark, C. A. 2013. Infection of sweetpotato by Fusarium solani and Macrophomina phaseolina prior to harvest. Plant Dis. 97:1636-1644.

Desjardins, A. E. 2006. Fusarium Mycotoxins: Chemistry, Genetics, and Biology. American Phytopathological Society, St. Paul, MN.

Doohan, F., Brennan, J., and Cooke, B. 2003. Influence of climatic factors on Fusarium species pathogenic to cereals. Pages 755-768 in: Epidemiology of Mycotoxin Producing Fungi. X. Xu, J. A. Bailey, and B. M. Cooke, eds. Springer, Dordrecht, The Netherlands.

Edmunds, B., Boyette, M., Clark, C., Ferrin, D., Smith, T., and Holmes, G. 2008. Postharvest Handling of Sweetpotatoes. North Carolina Cooperative Extension Service, Raleigh.

Ezell, B., Wilcox, M., and Demaree, K. 1956. Vegetable storage, physiological and biochemical effects of storage humidity on sweet potatoes. J. Agric. Food Chem. 4:640-644.

FAOSTAT. 2009. Statistical databases. Food and Agriculture Organization of the United Nations, Rome, Italy.

Farr, D. F., and Rossman, A. Y. 2006. Fungal databases, systematic mycology and microbiology laboratory. Online publication. United States Department of Agriculture-Agricultural Research Service. http://nt.ars-grin.gov/ fungaldatabases/

Gaffoor, I., Brown, D. W., Plattner, R., Proctor, R. H., Qi, W., and Trail, F. 2005. Functional analysis of the polyketide synthase genes in the filamentous fungus Gibberella zeae (anamorph Fusarium graminearum). Eukaryot. Cell 4:1926-1933.

Geiser, D. M., del Mar Jiménez-Gasco, M., Kang, S., Makalowska, I., Veeraraghavan, N., Ward, T. J., Zhang, N., Kuldau, G. A., and O'Donnell, K. 2004. FUSARIUM-ID v. 1.0: A DNA sequence database for identifying Fusarium. Pages 473-479 in: Molecular Diversity and PCR-Detection of Toxigenic Fusarium Species and Ochratoxigenic Fungi. G. Mulè, J. A. Bailey, B. M. Cooke, and A. Logrieco, eds. Springer, Dordrecht, The Netherlands.

Geiser, D. M., Pitt, J. I., and Taylor, J. W. 1998. Cryptic speciation and recombination in the aflatoxin-producing fungus Aspergillus flavus. Proc. Natl. Acad. Sci. USA 95:388-393.

Goktepe, F., Seijo, T., Deng, Z., Harbaugh, B. K., Peres, N. A., and McGovern, R. J. 2007. Toward breeding for resistance to Fusarium tuber rot in caladium: Inoculation technique and sources of resistance. HortScience 42: 1135-1139.
Granke, L., and Hausbeck, M. 2010. Effects of temperature, humidity, and wounding on development of Phytophthora rot of cucumber fruit. Plant Dis. 94:1417-1424.

Hanson, L., Schwager, S., and Loria, R. 1996. Sensitivity to thiabendazole in Fusarium species associated with dry rot of potato. Phytopathology 86: 378-384.

Holmes, G. J., and Stange, R. R. 2002. Influence of wound type and storage duration on susceptibility of sweetpotatoes to Rhizopus soft rot. Plant Dis. 86:345-348.

Iowa State University PTF. 2003. DNA Extraction from Maize Callus, Maize Leaf Tissue, or Soybean Leaf Tissue for PCR. Iowa State University Department of Agronomy, Ames, IA.

Johnson, T., Wilson, N., Worosz, M. R., Fields, D., and Bond, J. K. 2015. Commodity highlight: Sweet potatoes. Vegetables and Pulses Outlook VGS-355-SA1. Online publication. United States Department of AgricultureEconomic Research Service. http://www.ers.usda.gov/media/1834605/vgs-355sa1.pdf

Larkin, M. A., Blackshields, G., Brown, N. P., Chenna, R., McGettigan, P. A., McWilliam, H., Valentin, F., Wallace, I. M., Wilm, A., Lopez, R., Thompson, J. D., Gibson, T. J., and Higgins, D. G. 2007. Clustal W and Clustal X version 2.0. Bioinformatics 23:2947-2948.

Leach, S., and Webb, R. 1981. Resistance of selected potato cultivars and clones to Fusarium dry rot. Phytopathology 71:623-629.

Lui, L., and Kushalappa, A. 2002. Response surface models to predict potato tuber infection by Fusarium sambucinum from duration of wetness and temperature, and dry rot lesion expansion from storage time and temperature. Int. J. Food Microbiol. 76:19-25.

Madden, L. V., Hughes, G., and Van den Bosch, F. 2007. The Study of Plant Disease Epidemics. American Phytopathological Society, St. Paul, MN.

Marin, S., Sanchis, V., and Magan, N. 1995. Water activity, temperature, and pH effects on growth of Fusarium moniliforme and Fusarium proliferatum isolates from maize. Can. J. Microbiol. 41:1063-1070.

Miller, J. D., Young, J. C., and Trenholm, H. L. 1983. Fusarium toxins in field corn. I. Time course of fungal growth and production of deoxynivalenol and other mycotoxins. Can. J. Bot. 61:3080-3087.

Monacell, J. T., and Carbone, I. 2014. Mobyle SNAP workbench: A web-based analysis portal for population genetics and evolutionary genomics. Bioinformatics 30:1488-1490.

NCDA\&CS. 2015. Marketing North Carolina Sweetpotatoes. Federal-State Market News Service, Raleigh, NC.

Nei, M., and Kumar, S. 2000. Molecular Evolution and Phylogenetics. Oxford University Press, New York.

O’Donnell, K., Sutton, D. A., Fothergill, A., McCarthy, D., Rinaldi, M. G., Brandt, M. E., Zhang, N., and Geiser, D. M. 2008. Molecular phylogenetic diversity, multilocus haplotype nomenclature, and in vitro antifungal resistance within the Fusarium solani species complex. J. Clin. Microbiol. 46: 2477-2490.

Peckham, J. C., Mitchell, F. E., Jones, O. H., Jr., and Doupnik, B., Jr. 1972. Atypical interstitial pneumonia in cattle fed moldy sweet potatoes. J. Am. Vet. Med. Assoc. 160:169-172.

Rabie, C. J., Sydenham, E. W., Thiel, P. G., Lubben, A., and Marasas, W. F. 1986. T-2 toxin production by Fusarium acuminatum isolated from oats and barley. Appl. Environ. Microbiol. 52:594-596.

R Core Team. 2013. R: A Language and Environment for Statistical Computing. R Foundation for Statistical Computing, Vienna.

Reid, L., Nicol, R., Ouellet, T., Savard, M., Miller, J., Young, J., Stewart, D. W., and Schaafsma, A. W. 1999. Interaction of Fusarium graminearum and $F$. moniliforme in maize ears: Disease progress, fungal biomass, and mycotoxin accumulation. Phytopathology 89:1028-1037.

Ross, P. F., Nelson, P. E., Richard, J. L., Osweiler, G. D., Rice, L. G., Plattner, R. D., and Wilson, T. M. 1990. Production of fumonisins by Fusarium moniliforme and Fusarium proliferatum isolates associated with equine leukoencephalomalacia and a pulmonary edema syndrome in swine. Appl. Environ. Microbiol. 56:3225-3226.

Rossi, V., Ravanetti, A., Pattori, E., and Giosue, S. 2001. Influence of temperature and humidity on the infection of wheat spikes by some fungi causing Fusarium head blight. J. Plant Pathol. 83:189-198.

Scott, G. 1992. Sweet potatoes as animal feed in developing countries: Present patterns and future prospects. Pages 13-98 in: Roots, Tubers, Plantains and Bananas in Animal Feeding. D. Machin and S. Nyvold, eds. FAO, Rome.

Stamatakis, A., Ludwig, T., and Meier, H. 2005. RAxML-III: A fast program for maximum likelihood-based inference of large phylogenetic trees. Bioinformatics 21:456-463.

Tamura, K., Stecher, G., Peterson, D., Filipski, A., and Kumar, S. 2013. MEGA6: Molecular evolutionary genetics analysis version 6.0. Mol. Biol. Evol. 30:2725-2729.

Ueno, Y., Sawano, M., and Ishii, K. 1975. Production of trichothecene mycotoxins by Fusarium species in shake culture. Appl. Microbiol. 30:4-9. 
Watanabe, M., Yonezawa, T., Lee, K., Kumagai, S., Sugita-Konishi, Y., Goto, K., and Hara-Kudo, Y. 2011. Molecular phylogeny of the higher and lower taxonomy of the Fusarium genus and differences in the evolutionary histories of multiple genes. BMC Evol. Biol. 11:322.

Waterhouse, A. M., Procter, J. B., Martin, D. M., Clamp, M., and Barton, G. J. 2009. Jalview version 2-a multiple sequence alignment editor and analysis workbench. Bioinformatics 25:1189-1191.

White, T. J., Bruns, T., Lee, S., and Taylor, J. W. 1990. Amplification and direct sequencing of fungal ribosomal RNA genes for phylogenetics. Pages
315-322 in: PCR Protocols: A Guide to Methods and Applications. Academic Press, New York.

Whiting, E., Khan, A., and Gubler, W. 2001. Effect of temperature and water potential on survival and mycelial growth of Phaeomoniella chlamydospora and Phaeoacremonium spp. Plant Dis. 85:195-201.

Wilson, B. J. 1973. Toxicity of mold-damaged sweetpotatoes. Nutr. Rev. 31: 73-78.

Woolfe, J. A. 1992. Sweet Potato: An Untapped Food Resource. Cambridge University Press, New York. 